\title{
Cardio-Vascular Complications of Hydroxychloroquine Use: Can Impact COVID-19 Pandemic
}

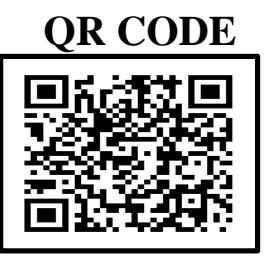

\section{TARUNA SINGH ${ }^{* 1}$, RAKSHIT ARORA², AMRIT SHARMA³}

As the coronavirus pandemic is on the rise, many compounds with anti-viral properties are under investigation.

Hydroxychloroquine (HCQ) being the daily debated daily during this COVID-19 pandemic is an immunomodulatory drug which has been used for indications like malaria, systemic lupus erythematosus and arthritis. Although some researchers have claimed its effectiveness against coronavirus, it results in proarrhythmic effects and drug-induced long QT syndrome. These cardiac issues while using hydroxychloroquine, have limited its use against coronavirus. A literature search was performed, and general safety information of this drug was collected. It can be concluded that this drug leads to cardiovascular events, heart disease, hypotension, tachycardia, and QT interval prolongation, sometimes in combination with other drugs and should be prescribed to the patients only after thoroughly estimating its benefit risk ratio.

KEYWORDS: Hydroxychloroquine, COVID-19, Coronavirus

\section{INTRODUCTION}

Hydroxychloroquine is an aminoquinolines that has received much attention during the current coronavirus pandemic. It is an approved drug for the treatment of systemic lupus erythematosus, rheumatoid arthritis and malaria, discoid lupus erythematosus, polymorphic light eczema and juvenile idiopathic arthritis. Contraindications to treatment with HCQ include existing retinopathy, visual field defects, porphyria, hypersensitivity to HCQ, auditory nerve damage and psoriasis. Hydroxychloroquine is well absorbed in oral administration. The half-life in the blood is very long, about 30-50 days. The degree of binding to proteins in plasma is relatively low, around 40 percent. It accumulates in certain tissues, including retina, muscles, and erythrocytes. Elimination takes place mainly via the kidneys.

Literature data suggests that it influences viral replication of SARS-CoV-2 $(1,2)$. In these studies, treatment regimens of 400 to $600 \mathrm{mg}$ of hydroxychloroquine/day for 5 to 10 days are suggested., Knowledge of toxicity and serious side effects mainly comes from retrospective studies and case reports. ${ }^{3}$ The randomized controlled trials that exist are small and have a narrow selection of participants, where patients with, for example, high age and comorbidities are often excluded. ${ }^{3}$ These factors make it difficult to know the exact frequency of serious side effects when using hydroxychloroquine in clinical practice.
In view of the potential massive use of hydroxychloroquine in the treatment of COVID-19, a review on safety of hydroxychloroquine was conducted. The relevant publications found were assessed by both the authors, and additional information in the literature references of each publication was sought.

Against COVID-19, HCQ has an indirect mechanism of action, related to the inflammatory response to the virus. Inhibition of inflammatory mediators such as the tumor necrosis factor alpha and its receptor, and interleukin 6 , causes discontinuation in the cascade of immune response to the virus, such as endothelial and alveolar patency, and therefore nearly two decades ago it was proposed that it would have a potential benefit in the prevention and treatment of acute respiratory distress caused by coronavirus. ${ }^{4}$

The literature describes interactions with betablockers with hepatic metabolism, amiodarone, and digoxin. 5 In patients with systemic lupus erythematosus, it was verified that CYP2D6 polymorphisms correlate with the serum drug levels, and this would be relevant in relation to the treatment of COVID-19: It is possible that patients treated with similar doses have disparate results according to the type of CYP2D6 polymorphism they have. (6) With the information available so far, some regulations on COVID-19 treatment with HCQ do not recommend the concomitant use of amiodarone and 
suggest monitoring both digoxin blood levels and the QT interval.

Meanwhile, in patients receiving HCQ, the combination of increasing doses of azithromycin increases the QT interval. This must be highlighted in view of the potential combined use of both drugs in current clinical trials for COVID-19.7,8

In a systematic review describing cardiovascular complications in patients with rheumatic diseases, disturbance in the conduction system, ventricular hypertrophy, alterations in left ventricular wall motion, symptomatic heart failure, pulmonary arterial hypertension, and valve dysfunction were reported on use of chloroquine and HCQ and the recovery rate after discontinuation was only around $45 \% .{ }^{9}$ Serious side effects that may occur as a result of a short period of treatment with hydroxychloroquine. One known long-term side effect is, retinopathy, which occurs as a result of tissue accumulation in melanin-containing cells.

A systematic review found publications of patients who on HCQ developed a specific cardiomyopathy, with granular inclusions with vacuolation of the cardiomyocytes, intravacuolar lamellar bodies, or curvilinear bodies. It is speculated that these lesions are attributed to a lysosomal effect, and some authors have described it as a phenocopy of Anderson-Fabry disease. Cardiomyopathy may cause hypertrophy and restrictive behavior. In most cases it manifests as clinical heart failure, or as alterations of the conduction system, and syncope. Approximately half of patients develop ventricular dsyfunction and mortality is $45 \%$. The chronic use of HCQ could then be the direct but extremely rare cause of an acquired lysosomal storage disease. ${ }^{10}$

In a review of electrocardiographic findings of patients with rheumatic diseases treated chronically with HCQ, normal PR, QRS, QTc values were observed, with only two cases of complete right bundle branch block and one case of left bundle branch block. However, this study has limitations, including not having baseline studies to make comparisons. ${ }^{11} \quad$ Tachycardia secondary to vasodilatation has been observed. Capel RA et al., in animal models, verified that HCQ causes bradycardia. ${ }^{12}$ However, in humans the heart rate could increase by indirect mechanisms. There are cases of HCQ-induced QT prolongation in the literature. hydroxychloroquine affects the repolarisation of myocardial cells and extend the QTC interval. This increases the risk of potentially lethal ventricular arrhythmias, such as Torsades de pointes. The prolongation of the QTc interval is dosedependent and usually occurs shortly after the first dose. ${ }^{13}$ It is important to consider the patient's overall risk factors for QTc prolongation and Torsade de pointes in treatment with hydroxychloroquine. Significant risk factors include high age, heart disease, certain electrolyte imbalances and concomitant treatment with multiple drugs that cause QTc prolongation. ${ }^{14,15}$ Most of these factors are also risk factors for severe COVID-19 infection. ${ }^{16} \mathrm{~A}$ non-randomised study reported that hydroxychloroquine in combination with azithromycin has an effect on COVID-19. ${ }^{2}$ Both hydroxychloroquine and azithromycin can prolong QTc. ${ }^{17}$ In a preliminary report, worrying QTc prolongations are reported in combination therapy with hydroxychloroquine and azithromycin for COVID-19. ${ }^{18}$

Hydroxychloroquine can rarely cause very serious skin reactions like Stevens-Johnson syndrome, toxic epidermal necrolysis and Drug reaction with eosinophilia and systemic symptoms. These conditions usually occur one to four weeks after starting treatment and can have a fatal outcome. ${ }^{19-21}$ Hydroxychloroquine may also result in outbreaks of psoriasis and should not be used in the presence of known psoriasis. Hydroxychloroquine can cause severe hypoglycaemia. ${ }^{22}$ The mechanism of hypoglycaemia is believed to be increased release of insulin from pancreatic beta cells as well as decreased degradation of insulin, which together causes hyperinsulinemic hypoglycaemia. ${ }^{23}$ In case of severe hypoglycaemia, treatment with 4-aminoquinolines should be discontinued immediately and glucose should be administered. Lastly, it is possible that when dealing with the massive use of HCQ cases of intoxication and overdose with these drugs may occur. In this sense, hypotension, conduction alterations and hypokalemia must be considered as primary findings.

\section{CONCLUSION}

Hydroxychloroquine have been proposed as a therapy candidate in COVID-19. Some patients suffer from serious side effects when treated with HCQ, such as hypotension, tachycardia, mild and asymptomatic QT prolongation, greater QT prolongation in 
concomitant treatment with azithromycin, interactions with amiodarone, digoxin, and betablockers, hypoglycemia and severe skin reactions. Ideally, it should only be used in accordance with approved indications or within clinical trials. More and larger randomized trials with adequate study protocols are required before any conclusions can be drawn on the efficacy and safety of COVID-19 therapy. It is still unclear when COVID-19 pandemic would end, and still a definite treatment is lacking. Therefore, the health care professionals do not have much management choices for coronavirus infection. So, hydroxychloroquine should be prescribed only after weighing the benefits and risks of this dug.

\section{REFERENCES}

1. Chen Z, Hu J, Zhang Z, et al. Efficacy of hydroxychloroquine in patients with COVID-19: results of a randomized clinical trial. Medrxiv. Epub 10

$$
\text { Apr }
$$

2020.

https://doi.org/10.1101/2020.03.22.20040758.

2. Gautret P, Lagier JC, Parola $P$, et al. Hydroxychloroquine and azithromycin as a treatment of COVID-19: results of an open-label nonrandomized clinical trial. Int J Antimicrob Agents. 2020;105949.

3. Ruiz-Irastorza G, Ramos-Casals M, Brito-Zeron P, et al. Clinical efficacy and side effects of antimalarials in systemic lupus erythematosus: a systematic review. Ann Rheum Dis. 2010;69(1):20-8.

4. Savarino A, Boelaert JR, Cassone A, Majori G, Cauda R. Effects of chloroquine on viral infections: an old drug against today's diseases? Lancet Infect Dis. 2003;3:722-7.

5. Projean $D$, Baune $B$, Farinotti $R$, et al. In vitro metabolism of chloroquine: identification of $\mathrm{CYP}_{2} \mathrm{C} 8$, $\mathrm{CYP}_{3} \mathrm{~A}_{4}$, and $\mathrm{CYP}_{2} \mathrm{D} 6$ as the main isoforms catalyzing N-desethylchlo $\neg$ roquine formation. Drug Metab Dispos. 2003;31:748-54.

6. Lee JY, Vinayagamoorthy $\mathrm{N}$, Han $\mathrm{K}$, et al. Association of polymorphisms of cytochrome $\mathrm{P} 450$ 2D6 with blood hydroxychloroquine levels in patients with systemic lupus erythematosus. Arthritis Rheumatol. 2016;68:184-90.

7. Hancox JC, Hasnain M, Vieweg WV, Crouse EL, Baranchuk A. Azithromycin, cardiovascular risks, QTc interval prolongation, torsade de pointes, and regulatory issues: a narrative review based on the study of case reports. Ther Adv Infect Dis. 2013;1:15565.
8. Ray WA, Murray KT, Hall K, Arbogast PG, Stein $\mathrm{CM}$. Azithromycin and the risk of cardiovascular death. N Engl J Med. 2012;366:1881-90.

9. Chatre C, Roubille F, Vernhet H, Jorgensen C, Pers YM. Cardiac complications attributed to chloroquine and hy $\neg$ droxychloroquine: a systematic review of the literature. Drug Saf. 2018;41:919-31.

10. Tselios K, Deeb M, Gladman DD, Harvey P, Urowitz MB. Antimalarial-induced cardiomyopathy: a systematic review of the literature. Lupus 2018;27:5919 .

11. Costedoat-Chalumeau N, Hulot JS, Amoura Z, et al. Heart conduction disorders related to antimalarials toxicity: an analysis of electrocardiograms in 85 patients treated with hydroxychloroquine for connective tissue diseases. Rheumatology 2007; 46: 8o8-10.

12. Capel RA, Herring $\mathrm{N}$, Kalla $\mathrm{M}$, et al. Hydroxychloroquine reduces heart rate by modulating the hyperpolarization-activated current If: Novel electrophysiological insights and therapeutic potential. Heart Rhythm 2015;12:2186-94.

13. Mzayek F, Deng H, Mather FJ, et al. Randomized dose-ranging controlled trial of AQ-13, a candidate antimalarial, and chloroquine in healthy volunteers. PLoS Clin Trials. 2007;2(1):e6.

14. Viskin S, Justo D, Halkin A, et al. Long QT syndrome caused by noncardiac drugs. Prog Cardiovasc Dis. 2003;45(5):415-27.

15. Tomaselli Muensterman E,Tisdale JE. Predictive analytics for identification of patients at risk for QT interval prolongation: a systematic review. Pharmacotherapy. 2018;38(8):813-21.

16. Yang J, Zheng Y, Gou X, et al. Prevalence of comorbidities in the novel Wuhan coronavirus (COVID-19) infection: a systematic review and metaanalysis. Int J Infect Dis. Epub 12 Mar 2020. https://doi.org/10.1016/j.ijid.2020.03.017.

17. Choi Y, Lim HS, Chung D, et al. Risk evaluation of azithromycin-induced QT prolongation in real-world practice. Biomed Res Int. 2018;2018:1574806.

18. Chorin E, Dai M, Shulman E, et al. The QT interval in patients with SARS-CoV-2 infection treated with hydroxychloroquine/ azithromycin. Medrxiv. Epub 3 apr 2020. https://doi.org/10.1101/2020.04.02.20047050. 19. Murphy M, Carmichael AJ. Fatal toxic epidermal necrolysis associated with hydroxychloroquine. Clin Exp Dermatol. 2001;26(5):457-8.

20. Cameron MC, Word AP, Dominguez A. Hydroxychloroquine induced fatal toxic epidermal 
necrolysis complicated by angioinvasive rhizopus. Dermatol Online J. 2014;20(11):13030/qt1q9oqoh5 21. Guillaume JC, Roujeau JC, Revuz J, et al. The culprit drugs in 87 cases of toxic epidermal necrolysis (Lyell's syndrome). Arch Dermatol. 1987;123(9):116670.

22. Johnson A, Eck LM. Hydroxychloroquineassociated hyperinsulinemic hypoglycemia [poster P3-471]. Endocr Rev. 2011;32.

23. Powrie JK, Smith GD, Shojaee-Moradie F, et al. Mode of action of chloroquine in patients with noninsulin-dependent diabetes mellitus. Am J Physiol.

1991; $260 \quad(6 \quad$ Pt $\quad 1)$ : $\begin{array}{lll}\text { E897- } 904 . & \end{array}$

Source of support: Nil, Conflict of interest: None declared

Cite this article as:

Singh T, Arora R, Sharma A. Cardio-Vascular Complications of Hydroxychloroquine Use:

Can Impact COVID-19 Pandemic. Int Healthc Res J. 2020;4(3):56-59.

Can Impact COVID-19 Pandemic
https://doi.org/10.26440/IHRJ/0403.06349

\section{AUTHOR AFFILIATIONS:}

1. MBBS, M.SC (Forensic Medicine), Private Practitioner and Consultant Doctor, New Delhi, India

2. MBBS, Private Practitioner, New Delhi

3. MDS (Oral Pathology), Consultant Dental Surgeon, Shiva Dental Clinic, Gajraula, Uttar Pradesh, India

Contact Corresponding Author at: tarunaforensic[at]gmail[dot]com 